\title{
Missions de l'éducation scientifique et technique
}

The tasks of scientific and technological education

Las misiones de la educación científica y tecnológica

Jean-Louis Martinand

\section{CpenEdition \\ Journals}

Édition électronique

URL : http://journals.openedition.org/ries/2550

DOI : $10.4000 /$ ries. 2550

ISSN : 2261-4265

Éditeur

Centre international d'études pédagogiques

Édition imprimée

Date de publication : 1 mars 2000

Pagination : 9-12

ISSN : $1254-4590$

Référence électronique

Jean-Louis Martinand, "Missions de l'éducation scientifique et technique », Revue internationale d'éducation de Sèvres [En ligne], 25 | 2000, mis en ligne le 01 mars 2003, consulté le 19 avril 2019 URL : http://journals.openedition.org/ries/2550 ; DOI : 10.4000/ries.2550 


\title{
Missions de l'éducation scientifique et technique
}

\author{
Jean-Louis Martinand \\ Professeur en sciences de l'éducation à l'École normale supérieure \\ de Cachan
}

Le but de cette intervention est de proposer quelques idées, peut-être provocatrices ou paradoxales, pour inciter au débat.

Je partirai d'une opinion, souvent acceptée comme un fait, selon laquelle nous devons faire face à ce qui est qualifié de "croissance exponentielle " des connaissances scientifiques et d' «accélération » des mutations techniques.

\section{Croissances et mutations exponentielles et accélérées}

Mon premier point consiste à soutenir que, si tel est le cas, alors cela remet en question toute l'éducation, tous les contenus de formation. Et on ne peut que s'étonner de certaines carences : ainsi les programmes français de $4^{\mathrm{e}} \mathrm{de}$ collège (14-15 ans) font une place importante à la science et à la technique du $\mathrm{XIX}^{\mathrm{e}}$ siècle pour la révolution industrielle et les changements culturels, mais ceux de $3^{\mathrm{e}}$ (15-16 ans) ne disent rien de cette croissance exponentielle et de cette accélération au $\mathrm{Xx}^{\mathrm{e}}$ siècle! De même, on trouve des textes littéraires, philosophiques et même scientifiques de savants dans les manuels de littérature jusqu'au XIX ${ }^{e}$ siècle, mais rien de tel au $\mathrm{XX}^{\mathrm{e}}$ siècle.

Si l'assertion de la croissance exponentielle et de l'accélération est vraie, cela exige de penser les contenus dans un équilibre global raisonnable. Il n'est pas possible de maintenir le grand écart que pratiquent certains en affirmant, d'un côté, que toute l'école élémentaire doit être recentrée sur la notion "lire/écrire/compter » et, de l'autre, que l'éducation scientifique, sous la forme d'actions comme « la main à la pâte », est prioritaire.

Le besoin de définir des propositions équilibrées fondées sur une appréciation objective et critique des évolutions appelle alors l'attention. C'est mon second point. Or la réalité, avec ses tendances, est bigarrée. Oui, nous vivons des mutations dans la connaissance et l'usage du vivant, dans la manipulation et la communication de l'information. Mais peut-on parler pour autant de croissance exponentielle des connaissances et d'accélération des mutations techniques? 
En termes de quantité d'informations stockées, diffusées, consultées, il y a sans doute croissance exponentielle. Mais deux remarques s'imposent : la première est que les développements exponentiels sont historiquement limités par saturation ou par mutation ; la seconde est que connaissance et information ne sont pas exactement la même chose. Y a-t-il croissance exponentielle des idées fondamentales ? Sommes-nous en état de révolution permanente?

Certes, nous sentons bien que l'éducation doit construire des connaissances flexibles, adaptatives. Mais cela ne rend pas caducs, pour beaucoup de domaines, les commencements et les bases qui y sont donnés, même s'ils doivent être refondus. Ensuite, la masse des connaissances factuelles, nouvelles et changeantes, à intégrer dans l'éducation n'est peut-être pas si importante partout.

Enfin, il y a contradiction à affirmer, d'un côté, que le rôle de l'enseignant ne sera plus tellement de transmettre des savoirs parce que ceux-ci changent trop vite et, de l'autre, que la technologie de l'information et de la communication va bouleverser les processus d'enseignement et d'apprentissage. Il faut manifestement ajuster l'analyse.

Et du côté des techniques? Il y a des changements évidents, soutenus, parfois très rapides, qui touchent le monde entier. Les organismes génétiquement modifiés se répandent en Amérique latine, les techniques contemporaines de génie civil, de télécommunication, de transport pénètrent l'Afrique... En réalité, partout dans le monde, les techniques « avancées » et " retardées » cohabitent.

Mais là encore, il faut prendre la mesure objective des tendances. Si les microprocesseurs changent vite, on ne peut qu'être frappé par la longévité des avions (jusqu'à trente ans) ou des moteurs automobiles. On doit aussi apprécier la manière dont, partout, les usagers adoptent, refusent ou détournent l'offre technique qui n'est jamais entièrement subie et qui peut, au contraire, susciter une créativité inattendue : les voitures ou les lave-linge ne sont pas les mêmes et ne donnent pas lieu aux mêmes utilisations de part et d'autre de l'Atlantique Nord.

Je voudrais souligner, enfin, que les croissances et les mutations exponentielles et accélérées se font d'abord avec les hommes. Leurs capacités sont souvent sous-estimées : qui aurait osé prédire, en 1900, qu'aujourd'hui en France toute la classe d'âge de 18 à 60 ans aurait son permis de conduire automobile et que les compétences qui en résultent ont facilité beaucoup d'adaptations à des mutations du travail professionnel ?

\section{Culture scientifique et culture technique}

Face à cette situation complexe, il s'agit aussi d'apprécier avec équilibre l'état des débats et des réflexions sur l'école. Mon troisième point veut rappeler les points de vue anciens et récents sur la culture scientifique et technique.

La culture scientifique est, certes, peu répandue lorsqu'on la rapporte à l'état de l'alphabétisation aujourd'hui et aux risques que ses carences peuvent 
faire courir à tous. Mais l'éducation scientifique est déjà ancienne. En France, l'obligation des leçons de choses date des années 1880 : il s'agissait d'enseigner " ce que nul ne peut ignorer » de la nature et de la technique et "d'apporter la chose » pour la faire voir. Et dans l'enseignement secondaire, la bataille des humanités modernes - sciences et langues vivantes - marque le passage du XIX ${ }^{e}$ $\mathrm{au} \mathrm{XX}^{\mathrm{e}}$ siècle. L'éducation scientifique doit sans doute être mise à jour, adaptée à l'enseignement de masse, elle doit se défendre contre réductions et dénaturations, mais elle existe.

La culture technique, quant à elle, est une idée très récente. Au moins pour l'école, car on connaît bien la vigueur des cultures techniques de métier ou d'affinité avec leurs technicités partagées, cultivées ou valorisées. Mais il s'agit ici de la culture technique dans la culture générale : cette idée a sans doute moins d'une génération - si on ne la confond pas avec la formation civique et morale qui a pu justifier l'éducation polytechnique dans les pays socialistes. Dans un projet de réforme célèbre comme le plan Langevin-Wallon, après la seconde guerre mondiale en France, on trouve la défense de l'égale dignité du travail, de la culture générale " moderne " dans la formation professionnelle, de l'intérêt de la technique si elle peut être présentée comme application des sciences. La pensée est celle de l'humanisme scientifique des années 1900 ou 1920. Il n'est pas question de culture technique pour tous.

Ainsi, il semble qu'il y a bien déficit de pensée sur la culture technique et l'éducation technologique et donc urgence à y réfléchir. Mais il ne faut pas les isoler ; le travail sur la question de la technique doit reprendre la question de la science. Dans tous les cas, il s'agit de poser le problème des savoirs ou, plutôt, des technicités qui doivent être partagées et valorisées pour tous comme composantes d'une culture générale de notre temps. Il s'agit aussi d'affronter les enjeux de citoyenneté qui sont inhérents aux développements et aux mises en œuvre des sciences et des techniques et qui posent les problèmes aigus des rapports aux savoirs et aux ignorances dans la démocratie, la vie domestique et la vie quotidienne.

\section{Missions et fondements}

Dans cet esprit, je voudrais formuler, en quatrième point, ce qui me paraît devoir être aujourd'hui les missions de l'éducation scientifique et technologique pour tous. Je souhaite parler de missions parce que la formation doit donner lieu à la fois à un débat d'ordre politique et à un projet d'ordre pédagogique ; parler d'emblée de finalités ou de compétences risquerait de faire dériver la discussion vers des abstractions consensuelles et sans conséquence, car banales et cumulatives (les finalités), ou vers des définitions techniques réductrices et prématurées (les objectifs).

Je distinguerai quatre missions fondamentales :

- donner une familiarité pratique avec des objets, des processus, des 
procédés : construire, en tenant compte de la diversité et de l'hétérogénéité des contextes et des élèves, une référence empirique commune, complétant et compensant celle du milieu familial et des médias ;

- faire prendre la mesure de la diversité du monde naturel et technique : appréhender la richesse extrême des « règnes » naturels et artificiels et donner les outils d'une lecture compréhensive du monde, impliquant une reprise de la réflexion sur le caractère nécessairement « encyclopédiste » et " paradoxal » mais aussi raisonnablement ambitieux de cette éducation ;

- populariser le patrimoine universel de représentations, d'explications, d'inventions accumulées, validées, avec les éléments de langages spécifiques qui leur sont associés ;

- permettre "l'interpellation " des experts et des argumentations, la délibération dans les décisions et les anticipations collectives.

Pour finir, en cinquième point, et dans la perspective esquissée par ces quatre missions, j'aimerais mettre l'accent sur deux fondements, pour une pensée évolutive, qui relèvent avant tout de l'éducation scientifique et technologique :

- le rapport expérimental aux processus naturels et artificiels, allant audelà de l'observation pour manipuler les variables et constituer une technique des effets ;

- la pensée avec des modèles, ce qui ne revient pas à apprendre des modèles, mais à adapter, utiliser, bricoler des modèles d'interprétation de la nature et de la technique, à la fois opérationnels et hypothétiques.

Je sais tout ce que ces deux propositions, prises dans leur sens précis et fort, peuvent avoir de discutable et de provocateur - au regard des moyens à mettre en œuvre, des compétences des maîtres ou des conceptions dominantes dans la culture. Mais nul ne peut nier qu'elles correspondent à ce qui est au cœur du dynamisme de la connaissance scientifique et de l'innovation technique. Discuter des "fondamentaux " c'est aussi discuter du rapport expérimental à la réalité et de son interprétation par modèles. 